\title{
New Results on the Moments of the Eigenvalues of the Schrödinger Hamiltonian and Applications
}

\author{
A. Martin
}

Theory Division, CERN, CH-1211 Geneva 23, Switzerland

\begin{abstract}
We show that the moments of order $\gamma$ of the eigenvalues of the Schrödinger Hamiltonian in $n$ dimensions can be related to moments of order less than or equal to $\gamma-\frac{1}{2}$ in $n+1$ dimensions. This makes it possible to improve the bounds on the sum of the eigenvalues in three dimensions and consequently the Lieb-Thirring bound on the binding energy of matter.
\end{abstract}

\section{Introduction}

A technical, but essential ingredient of the proof of "stability" of matter by Lieb and Thirring [1] is a bound on the sum of the eigenvalues of a one-particle Hamiltonian in three space dimensions. The Lieb-Thirring bound has the form

$$
E>-C N\left[1+\left(\frac{\sum_{j=1}^{p} Z_{j}^{7 / 3}}{N}\right)^{1 / 2}\right]^{2} \alpha^{2} m
$$

for a system with $N$ electrons and point nuclei of charges $Z_{1}, Z_{2}, \ldots, Z_{p} ; \alpha$ is the fine structure constant and $m$ the electron mass.

The constant $C$ is proportional to the power $2 / 3$ of the coefficient appearing in the inequality

$$
\sum\left|e_{i}\right|<L_{1,3} \int\left|V^{-}\right|^{5 / 2} d^{3} x
$$

where the $e_{i}$ 's are the negative eigenvalues of the three-dimensional one-particle Schrödinger equation with a potential $V$ (for the notation $L_{1,3}$ see Sect. 2). In [1], Lieb and Thirring get

$$
L_{1,3} \leqq \frac{4}{15 \pi}
$$

and correspondingly

$$
C=4.16 \text {. }
$$


The best possible value one can hope to get for $L_{1,3}$ is given by the semiclassical estimate [2]

$$
L_{1,3} \cong \frac{1}{15 \pi^{2}},
$$

which is better by a factor $4 \pi$. If Eq. (5) was a bound, this would lead to the best possible $C$,

$$
C \simeq 0.77 \text {, }
$$

which is what one gets if one takes isolated atoms and assumes the validity of the Thomas-Fermi approximation. The Thomas-Fermi approximation is known to hold in the limit of large $Z$ [3].

Shortly after the Lieb-Thirring result, Lieb [4] found a bound on the total number of bound states up to a given energy $E$, in three dimensions:

$$
N_{3}(E)<0.116 \int d^{3} x\left|(V-E)_{-}\right|^{3 / 2} \text {. }
$$

By integration this gives a bound on $\sum\left|e_{i}\right|$ which is

and this leads to

$$
\sum\left|e_{i}\right|<\frac{1}{1.82} \frac{4}{15 \pi} \int d^{3} x\left|V_{-}\right|^{5 / 2},
$$

$$
C=2.78 \text {. }
$$

The purpose of this paper is to show a link between moments in various space dimensions which allows, among other things, a slight improvement of inequality $(8)$, and has an intrinsic interest.

\section{Comparison of Moments in Various Space Dimensions}

In what follows, we shall take the notation of [5]. We have

$$
\sum\left|e_{i}\right|^{\gamma} \leqq L_{\gamma, n} \int\left|V_{-}\right|^{\frac{n}{2}+\gamma} d^{n} x,
$$

where $L_{\gamma, n}$ is the smallest constant for which (10) holds, $n$ is the number of space dimensions, $\gamma$ is the order of the moment, the $e_{i}$ 's are the negative eigenvalues and $V_{-}$is the negative part of $V$. Notice that $L_{0,1}$ and $L_{0,2}$ are infinite.

If $V=\lambda v$, and if we let $\lambda$ go to infinity we get the semiclassical limit [2,5],

$$
\sum\left|e_{i}\right|^{\gamma} \simeq L_{\gamma, n}^{c} \lambda^{\frac{n}{2}+\gamma} \int\left|v_{-}\right| \frac{n}{2}{ }^{+\gamma} d^{n} x .
$$

We have

$$
L_{\gamma, n}^{c}=2^{n} \pi^{-n / 2} \frac{\Gamma(\gamma+1)}{\Gamma\left(\gamma+1+\frac{n}{2}\right)} .
$$

There is one case where we know that $L_{\gamma, n}=L_{\gamma, n}^{c}$, which is $n=1, \gamma=3 / 2$, and by an argument of Aizenman and Lieb [6] $n=1$ and any $\gamma>3 / 2$. In the case of the number of bound states, $\gamma=0$, we know from the explicit examples of Glaser, Grosse, and Martin [7] that

$$
L_{0, n}>L_{0, n}^{c}, \quad \forall n .
$$


For $\gamma=1$ (i.e., the sum of the energies) and three dimensions, there is no counterexample to the conjecture that the semiclassical constant is in fact the strict bound.

To establish a link between moments in dimensions $n$ and $n+1$, we shall use the fact that inequality (10) holds for a completely arbitrary potential. We call $\mathbf{x}=x_{1}, x_{2}, \ldots, x_{n}$ the first $n$ co-ordinates and $t$ the last one in $n+1$ dimensions.

We shall begin by an example. Take

$$
V(\mathbf{x}, t)=v(\mathbf{x})+\lambda t^{2} .
$$

Then the total number of negative energy bound states of $V$ is given by

$$
N_{n+1}(V) \leqq L_{0, n+1} \int d x^{n} d t\left|\left[v(\mathbf{x})+\lambda t^{2}\right]_{-}\right|^{\frac{n+1}{2}},
$$

where $N_{p} \equiv L_{0, p}$ designates the total number of bound states in $p$ dimensions. The integral over $t$ can be carried exactly. It gives

$$
N_{n+1}(V) \leqq L_{0, n+1} \frac{\Gamma\left(\frac{n}{2}+\frac{3}{2}\right)}{\Gamma\left(\frac{n}{2}+2\right)} \frac{\sqrt{\pi}}{\sqrt{\lambda}} \int\left|v_{-}\right|^{\frac{n}{2}+1} .
$$

Let us now analyze what $N$ is. If the pair $\left(E_{i}, \psi_{i}(x)\right)$ is the bound-state energy and the wave function of a state in potential $v$, and $\left(\varepsilon_{k}, \phi_{k}(t)\right)$ is one of the energy levels of the harmonic oscillator $\lambda t^{2}$, the levels of $V$ are given by

$$
\left(E_{i}+\varepsilon_{k}, \psi_{i}(x) \phi_{k}(t)\right) \text {. }
$$

In $N$ we count the energy levels $E_{i}+\sqrt{\lambda}, E_{i}+3 \sqrt{\lambda}, \ldots, E_{i}+(2 n+1) \sqrt{\lambda}$, as long as $E_{i}+\varepsilon_{k}$ is negative. Therefore the level $E_{i}$ will be counted approximately (neglecting the zero-energy contribution)

$$
\left|E_{i}\right| /(2 \sqrt{\lambda}) \text { times. }
$$

So we get

$$
\frac{\sum\left|E_{i}\right|}{2 \sqrt{\lambda}} \lesssim \sqrt{\pi} \frac{\Gamma\left(\frac{n}{2}+\frac{3}{2}\right)}{\Gamma\left(\frac{n}{2}+2\right)} \frac{L_{0, n+1}}{\sqrt{\lambda}} \int\left|v_{-}\right|^{\frac{n}{2}+1} d^{n} x,
$$

and, therefore, letting $\lambda$ go to zero,

$$
L_{1, n} \leqq 2 \sqrt{\pi} \frac{\Gamma\left(\frac{n}{2}+\frac{3}{2}\right)}{\Gamma\left(\frac{n}{2}+2\right)} L_{0, n+1}
$$

It is easy to check, from Eq. (12), that we have

$$
L_{1, n} \leqq \frac{L_{1, n}^{c}}{L_{0, n+1}^{c}} L_{0, n+1} .
$$


In fact, this result was to be expected without calculation for the following reason. Instead of starting from the potential $V=v+\lambda t^{2}$, we could have taken $g V$, letting $g$ go to infinity. Then

$$
N_{n+1} \simeq L_{0, n+1}^{c} g^{\frac{n+1}{2}} \int\left|V_{-}\right|^{\frac{n+1}{2}} d^{n} x d t
$$

and, in $n$ dimensions:

$$
\sum\left|E_{i}\right| \simeq L_{1, n}^{c} g^{\frac{n}{2}+1} \int\left|v_{-}\right|^{\frac{n}{2}+1} d^{n} x
$$

so that unavoidably the ratio of $\sum\left|E_{i}\right|$ and $N$ contains the semiclassical constants.

There are two possible generalizations of this result. First, starting from dimension $n+1$, one can replace the harmonic oscillator potential by another one. If the energy levels $\varepsilon_{k}$ increase according to a power law $\varepsilon_{k} \sim c k^{\alpha}$, we shall get a different moment of the eigenvalues $\left|E_{i}\right|$ of the $n^{\text {th }}$ dimensional potential. Only the asymptotic behaviour for large $k$ of the $\varepsilon_{k}$ 's matters, because we let the strength of the auxiliary potential go to zero. So, to get moments of the eigenvalues of the $n$-dimensional potential, it is sufficient to consider power potentials. For $V=|t|^{v}$, the eigenvalues $\varepsilon_{k}$ behave like const $\times k^{2 v /(v+2)}$ [8], and one gets a bound on the moment of order $\gamma=(v+2) / 2 v$. Notice that by this method it is impossible to obtain moments of order less than $\frac{1}{2}$. This is due to the fact that in any one-dimensional potential, the $n^{\text {th }}$ energy level has an energy which is less than const $\times n^{2}$. To prove this, notice that

$$
V(x)<\sup _{a<y<b} V(x) \text { for } a<x<b,+\infty \text { for } x \text { outside } a b,
$$

and use the monotonicity of the energy levels with respect to the potential.

By using the trick of the large coupling constant we get, without calculation:

$$
L_{\gamma, n} \leqq \frac{L_{\gamma, n}^{c}}{L_{0, n+1}^{c}} L_{0, n+1} \quad \text { for } \quad \gamma \geqq 1 / 2 .
$$

This can be generalized still further. One can start from the inequality for a given moment different from zero in $(n+1)$ dimensions, in a potential $v(x)+\lambda t^{v}$. Then the moment of order $\gamma$ is

with $\varepsilon_{k} \sim$ const $\times k^{2 v /(v+2)}$. So

$$
\sum_{i, k}\left|\left(E_{i}+\varepsilon_{k}\right)_{-}\right|^{\gamma}
$$

$$
\sum_{k}\left|\left(E_{i}+\varepsilon_{k}\right)_{-}\right|^{\gamma} \simeq \text { const }\left|E_{i}\right|^{\gamma+\frac{2 v}{v+2}} .
$$

Again, explicit calculations are not needed because of the large coupling constant argument, and we get finally

$$
L_{\delta, n} \leqq \frac{L_{\delta, n}^{c}}{L_{\gamma, n+1}^{c}} L_{\gamma, n+1} \quad \text { with } \quad \delta \geqq \gamma+1 / 2 .
$$

This inequality gives a constructive proof of some results in low dimensions.

i) The existence of a bound on the number of bound states for three dimensions implies the existence of a bound on the moments of order $\frac{1}{2}$ in two dimensions and on the sum of the energies in one dimension. 
ii) The existence of a bound for $n=2, \gamma>0$ implies the existence of a bound for $n=1, \gamma>\frac{1}{2}$.

iii) If we know (at present, we don't!) that $L_{\gamma, n}=L_{\gamma, n}^{c}$, then

$$
L_{\gamma^{\prime}+\frac{1}{2}, n-1}=L_{\gamma^{\prime}+\frac{1}{2}, n-1}^{c} \text { for } \gamma^{\prime} \geqq \gamma .
$$

In $[5,9]$, another quantity was introduced, $L_{\gamma, n}^{1}$, which replaces $L_{\gamma, n}$ in (10) when there is only one bound state, or if one uses only the lowest bound state:

$$
\begin{gathered}
\left|e_{0}\right|^{\gamma} \leqq L_{\gamma, n}^{1} \int\left|V_{-}\right|^{\frac{n}{2}+\gamma}, \\
L_{0, n}^{1}=[\pi n(n-2)]^{-n / 2} \Gamma(n) / \Gamma(n / 2) .
\end{gathered}
$$

Again in [5], the conjecture was made that

$$
L_{\gamma, n}<\sup \left(L_{\gamma, n}^{c}, L_{\gamma, n}^{1}\right) \text {. }
$$

This conjecture was later shown to be incorrect in large-dimensional spaces for $n \geqq 7$ by Glaser, Grosse, and Martin [7]. However, there is no counter-example to this conjecture for $n=1,2,3$. Then one can define $\gamma_{c, n}$ for which $L_{\gamma, n}^{1}=L_{\gamma, n}^{c}$. From (22), one then deduces

$$
\gamma_{c, 1} \leqq \frac{1}{2}+\gamma_{c, 2}, \quad \gamma_{c, 2} \leqq \frac{1}{2}+\gamma_{c, 3} .
$$

According to Barnes, this is indeed true numerically [5]: $\gamma_{c, 1}=1.5, \gamma_{c, 2} \cong 1.165, \gamma_{c, 3}$ $\cong 0.863$.

\section{The Case of Few Bound States}

In the previous section, we introduced $L_{\gamma, n}^{1}$, the smallest possible constant in inequality (11), corresponding to the lowest (and possibly unique) negative energy bound state. In particular, if there is at least one bound state we have

$$
1 \leqq L_{0, n}^{1} \int\left|V_{-}\right|^{n / 2} d^{n} x,
$$

and, if there are two bound states [9], we have

$$
2 \leqq L_{0, n}^{1} \int\left|V_{-}\right|^{n / 2} d^{n} x .
$$

Indeed, the higher bound state has a wave function which can be separated into two nodal regions because, from orthogonality to the ground state, it has necessarily positive and negative values, and it has been shown by Courant and Hilbert that the $k^{\text {th }}$ wave function has at most $k$ nodal regions. Applying (24) to the two nodal regions of the first excited state, we get (25).

Now we can apply the same strategy as in the previous section. Starting from a potential in $n+1$ dimensions $V=v(x)+\lambda t^{2}$, but, instead of letting $\lambda$ eventually go to zero, choose $\lambda$ in such a way that $V$ has only one or only two bound states.

Since the lowest level of the harmonic oscillator is at $\varepsilon=\sqrt{\lambda}$, we shall take first $\sqrt{\lambda}=-E_{0}-\eta$, where $E_{0}$ is the ground state of $v$, guaranteed to be non-degenerate, and $\eta$ is positive and sufficiently small. Then $V$ has only one bound state and we get

$$
\left|E_{0}\right|<\frac{1}{2} \frac{L_{1, n}^{c}}{L_{0, n+1}^{c}} L_{0, n+1}^{1} \int\left|v_{-}\right|^{\frac{n}{2}+1} d^{n} x
$$


Notice the factor $\frac{1}{2}$ coming from the fact that the ground state of a harmonic oscillator has only half a quantum, while the spacing, occurring in the previous section, is a quantum. Inequality (26) can be rewritten as

$$
L_{1, n}^{1}<\frac{1}{2} \frac{L_{1, n}^{c} L_{0, n+1}^{1}}{L_{0, n+1}^{c}}=\frac{\sqrt{\pi}}{2} \frac{\Gamma(n+2)}{\Gamma\left(\frac{n}{2}+2\right)\left[\pi\left(n^{2}-1\right)\right]^{\frac{n+1}{2}}}
$$

For $n=3$, this gives

$$
L_{1,3}^{1}<\frac{1}{10 \pi^{2}}<0.0102
$$

This is to be compared to Barnes's numerical calculation [5]

$$
L_{1,3}^{1} \simeq 0.0063 \text {. }
$$

From Eq. (27), we conclude

$$
L_{1, n}^{1} / L_{1, n}^{c}<1 \text { for } n \geqq 5 \text {. }
$$

Let us now look at $E_{1}$, the first excited state of $v$, using Eq. (25). If we take $\sqrt{\lambda}=\left|E_{1}\right|-\eta, V$ has $t w o$ bound states and we get

$$
\left|E_{1}\right|<\frac{1}{4} \frac{L_{1, n}^{c}}{L_{0, n+1}^{c}} L_{0, n+1}^{1} \int\left|v_{-}\right|^{\frac{n}{2}+1} d^{n} x
$$

Notice that already for $n=3$, the coefficient in (29) is 0.0051 , i.e., less than the "exact" coefficient obtained by Barnes for $\left|E_{0}\right|$.

More generally, we get for the $p+1$ first bound states,

$$
\left|E_{0}\right|+\left|E_{1}\right|+\ldots+\left|E_{p}\right|<\frac{p+2}{4} \sqrt{\pi} \frac{\Gamma(n+2)}{\Gamma\left(\frac{n}{2}+2\right)\left[\pi\left(n^{2}-1\right)\right]^{\frac{n+1}{2}}} \int\left|v_{-}\right|^{\frac{n}{2}+1} d^{n} x .
$$

It is easy to see that for any given $p$, one can manage to have the coefficient in (30) less than the semiclassical constant by taking $n$ large enough. For instance, for the first three bound states, this happens for $n \geqq 7$. This supports the conjecture that for $n$ large enough it is the semiclassical constant which gives the bound, but it is also compatible with a situation similar to what happens for the number of bound states in a spherically symmetric potential, as shown in [7]: for $n \geqq 7$ the configurations, presumably saturating the bound, obtained by a variational approach, have more and more bound states as $n$ increases.

Naturally, it is clear that all we have said about $\left|E_{0}\right|,\left|E_{1}\right|$, etc., can be repeated for moments $\left|E_{0}\right|^{\gamma},\left|E_{1}\right|^{\gamma}$, etc., with $\gamma \geqq \frac{1}{2}$. The bounds will be in terms of $L_{0, n+1}^{1}$, $L_{0, n+1}^{c}, L_{\gamma, n}^{c}$. We leave this as an exercise to the reader.

\section{Applications to the Problem of Stability of Matter, and Conclusions}

As we said in the introduction, the constant entering into the lower bound (1) of the binding energy of matter is linked to the value of $L_{1,3}$ in the approach of Lieb and 
Thirring [1]. The best value for $L_{1,3}$ has, up to now, been obtained from the Lieb bound on $N_{3}=L_{0,3}$ :

$$
L_{1,3}<0.0467 \text {. }
$$

From Sect. 2 we have a bound on $L_{1,3}$ in terms of $L_{0,4}$ and also $L_{0,5}$, since moments can increase by only half a unit when the dimension of the space decreases by one unit:

$$
L_{1,3}<\frac{32}{15} L_{0,4}, \quad L_{1,3}<4 \pi L_{0,5} .
$$

For $L_{0,4}$ and $L_{0,5}$ we can use the bounds by Lieb [4] or by Blanchard, Rezende, and Stubbe [10]. The values of Lieb are the best. They are given by

$$
\begin{aligned}
& L_{0, n}<\inf (2 \pi)^{-n / 2} \int_{0}^{\infty} s^{-1-\frac{n}{2}} f(s) d s \\
& f(s) \text { convex } \\
& 1=\int_{0}^{\infty} \frac{e^{-y}}{y} f(2 y) d y .
\end{aligned}
$$

It is easy to prove that the best choice for $f$ is $f=0$ for $s<s_{0}, f=\operatorname{const}\left(s-s_{0}\right)$ for $s \geqq s_{0}$. In this way one gets

$$
L_{0,4}<0.0191, \quad L_{0,5}<0.00321,
$$

and respectively

$$
L_{1,3}<0.0407, \quad L_{1,3}<0.0404 .
$$

Comparing with (31), we see that we get a rather minor improvement. The corresponding value of $C$, entering into (1), becomes $C=2.52$, to be compared with $C=2.78$.

Now the question is to know if one can hope to do better. With the present method, we cannot hope to find the best possible answer, which would be $L_{1,3}=L_{1,3}^{c}$ because, from Eq. (20), this would be possible only if $L_{0,5}$ was equal to the classical value. This is not the case. We know that $L_{0,5} \geqq L_{0,5}^{1}>L_{0,5}^{c}$. In fact, it is unfortunate that this will never happen in any number of dimensions because of inequality (13). So our method will not, in its present form, allow us to prove that $C$ is equal to the Thomas-Fermi value.

This being said, there is room for improvement if progress is made on $L_{0,4}$ and $L_{0,5}$. In the spherically symmetric case, we know the best possible value for $L_{0,4}$. It is [7]

and hence this would give

$$
L_{0,4}=\frac{3}{32 \pi^{2}} \simeq 0.00950,
$$

$$
L_{1,3}=0.0203 \text { and } C=1.593 \text {. }
$$

Unfortunately, $V$ is not spherically symmetric, even if $v$ is taken to be spherically symmetric. One would need some argument to prove that the extremum for $L_{0,4}$ is reached for a spherically symmetric potential in four dimensions. Then, if this is so, one realizes, following carefully the derivation of [7], that this potential would furthermore be unavoidably a potential containing only one bound state. 
There is also a different path followed by Thirring [11], showing that for very large $Z$ 's the leading contribution in the lower bound on the ground state energy indeed contains the Thomas-Fermi constant, but the remainder should be bounded and the simplicity of Eq. (1) is lost. So it is worth trying to improve the bound on the sum of the energies. This bound remained stationary during 12 years. Let us hope that it will take less time to get the best possible value.

Acknowledgements. It was in the stimulating atmosphere of discussions with H. Grosse, J. Stubbe, and L. Vasquez that I undertook this work. I am very grateful to them.

\section{References}

1. Lieb, E.H., Thirring, W.: Phys. Rev. Lett. 35, 687 (1975)

2. Martin, A.: Helv. Phys. Acta 45, 140 (1972);

Tamura, H.: Proc. Japan Acad. 50, 19 (1974)

3. Lieb, E.H., Simon, B.: Adv. Math. 23, 22 (1977)

4. Lieb, E.H.: Bull. Am. Math. Soc. 82, 751 (1976), Proceedings of Symposia in Pure Mathematics 36, 241 (1980), Commun. Math. Phys. 92, 473 (1984);

See also: Simon, B.: Functional integration and quantum physics, p. 95. New York: Academic Press 1979

5. Lieb, E.H., Thirring, W.: Studies in mathematical physics, Lieb, E.H., Simon, B., Wightman, A.S. (eds.), p. 269. Princeton, NJ: Princeton University Press 1976

6. Aizenman, M., Lieb, E.H.: Phys. Lett. 66A, 427 (1978)

7. Glaser, V., Grosse, H., Martin, A.: Commun. Math. Phys. 59, 197 (1978)

8. See, for instance: Quigg, C., Rosner, J.L.: Phys. Rep. 56, 167 (1979);

For a rigorous derivation, see for instance: Clark, C.: Siam Review 9, 627 (1967)

9. Glaser, V., Grosse, H., Martin, A., Thirring, W.: [5], p. 169

10. Blanchard, Ph., Rezende, F., Stubbe, J.: Lett. Math. Phys. 14, 215 (1987)

11. Thirring, W.: Commun. Math. Phys. 79, 1 (1981)

Communicated by A. Jaffe

Received February 9, 1989; in revised form April 19, 1989 